\title{
PSEUDOCOMPACTNESS AND CLOSED SUBSETS OF PRODUCTS
}

\author{
JAMES E. JOSEPH
}

\begin{abstract}
This paper contains several new characterizations of arbitrary pseudocompact spaces, i.e. spaces characterized by the property that all continuous real-valued functions on the space are bounded. These characterizations parallel known characterizations of Hausdorff spaces including the useful and well-known result that a space $Y$ is Hausdorff if and only if $\phi=\alpha$ whenever $\phi$ and $\alpha$ are continuous functions on a common domain into $Y$ which agree on a dense subset of the domain.
\end{abstract}

1. Introduction. Pseudocompact spaces which are not necessarily completely regular have been studied in $[\mathbf{J 1}]$ and [S], and some results have been produced that apply to noncompletely regular pseudocompact spaces in [BCM], [G2] and [SS]. In general, completely regular pseudocompact spaces have been more extensively studied (e.g., see [BCM], [C] $[\mathbf{G 2}],[\mathbf{H}]$, [SS], and more recently, $[\mathbf{T}])$. In this article we give several new characterizations of arbitrary pseudocompact spaces. These characterizations are interesting as analogues of well-known characterizations of Hausdorff spaces and one of them may be used to establish that certain functions into pseudocompact spaces are completely determined by their values on dense subsets of their domains.

2. Preliminaries. The closure of a subset $K$ of a space $X$ will be denoted by $\operatorname{cl}(K)$ and the family of open sets about $x \in X$ will be denoted by $\Sigma(x)$. If $\phi$, $\alpha: X \rightarrow Z$ and $\lambda: Y \rightarrow Z$ are functions, $\mathcal{E}(\phi, \lambda, X \times Y, Z)$ and $E(\phi, \alpha, X, Z)$ will represent $\{(a, b) \in X \times Y: \phi(a)=\lambda(b)\}$ and $\{x \in X: \phi(x)=\alpha(x)\}$, respectively. We will represent $\{(x, x): x \in X\}$ by $\Delta$, and the class of continuous real-valued functions on $Z$ by $C(Z)$. $R$ will denote the space of reals.

2.1. Definition. A point $z$ in a space $Z$ is in the closure of a subset $K$ of the space with respect to $g \in C(Z)(z \in \operatorname{cl}(K)(\bmod g))$ if $K \cap g^{-1}(H) \neq \varnothing$ for each $H \in \Sigma(g(z))$ in $R ; z$ is in the adherence of a filterbase $\Omega$ on $Z$ with respect to $g \in C(Z)(z \in \operatorname{ad} \Omega(\bmod g))$ if $z \in \operatorname{cl}(F)(\bmod g)$ for each $F \in \Omega$.

It is easily seen that $z \in \operatorname{cl}(K)(\bmod g)$ if and only if $g(z) \in \operatorname{cl}(g(K))$ in $R$ and hence that $z \in \operatorname{ad} \Omega(\bmod g)$ if and only if $g(z) \in \operatorname{ad} g(\Omega)$ in $R$. From these observations our first theorem is seen to be a paraphrase of the

Received by the editors June 23, 1977 and, in revised form, April 24, 1978.

AMS (MOS) subject classifications (1970). Primary 54D30, 54C30.

Key words and phrases. Pseudocompactness, filterbases, graphs. 
elementary and well-known result that $Z$ is pseudocompact if and only if $g(Z)$ is compact (or countably compact) for each $g \in C(Z)$.

2.2. THEOREM. The following statements are equivalent for a space $Z$ :

(a) $Z$ is pseudocompact;

(b) ad $\Omega(\bmod g) \neq \varnothing$ for each filterbase $\Omega$ on $Z$ and $g \in C(Z)$;

(c) ad $\Omega(\bmod g) \neq \varnothing$ for each countable filterbase $\Omega$ on $Z$ and $g \in C(Z)$.

We recall that a function $\phi$ from a space $X$ to a space $Z$ has a closed graph if the graph of $\phi, \mathcal{G}(\phi)$, is a closed subset of the product space $X \times Z$. The following theorem is proved in [J2]. The characterization in this theorem motivates Definition 2.4.

2.3. TheOrem. $A$ function $\phi: X \rightarrow Z$ has a closed graph if and only if: (1) $\{\phi(x)\}$ is closed in $Z$ for each $x \in X$, and (2) ad $\phi(\Omega) \subset\{\phi(x)\}$ for each $x \in X$ and filterbase $\Omega$ on $X-\{x\}$ with $\Omega \rightarrow x$.

2.4. Definition. A function $\phi: X \rightarrow Z$ has a strongly-subclosed graph with respect to $g \in C(Z)(\mathcal{G}(\phi)$ is strongly-subclosed $(\bmod g))$ if ad $\phi(\Omega)(\bmod$ $g) \subset\{\phi(x)\}$ for each $x \in X$ and filterbase $\Omega$ on $X-\{x\}$ with $\Omega \rightarrow x$.

From the statement in the first sentence of the paragraph preceding Theorem 2.2, we see easily that the following result holds.

2.5. Theorem. Let $\phi: X \rightarrow Z$ be a function and $g \in C(Z)$. Then $\mathcal{G}(\phi)$ is strongly-subclosed $(\bmod g)$ if and only if, for each $x \in X$ and filterbase $\Omega$ on $X-\{x\}$ converging to $x, g^{-1}(\operatorname{ad} g(\phi(\Omega))) \subset\{\phi(x)\}$.

By combining the results in Theorems 2.3 and 2.5, we observe that if $\mathcal{G}(\phi)$ is strongly-subclosed $(\bmod g)$ then $\mathcal{G}(g \circ \phi)$ is closed. The following example shows that the converse of this statement is not (in general) true.

2.6. ExAmple. Let $\phi, g:[0,1] \rightarrow R$ be defined by $\phi(x)=0$ for all $x$ and $g(x)=1$ for all $x$. Then $\mathcal{G}(g \circ \phi)$ is closed, but $\phi$ is not strongly-subclosed $(\bmod g)$. For example, let $F(n)=\{1 / k: k=n, n+1, \ldots\}$; then $\Omega=$ $\{F(1), F(2), \ldots\}$ is a filterbase on $[0,1]-\{0\}$ and $\Omega \rightarrow 0$; however, $[0,1] \subset$ ad $\phi(\Omega)(\bmod g)$.

3. The main results. In the following let $K$ denote a homeomorph of the subspace $\{0,1,1 / 2, \ldots, 1 / n, \ldots\}$ of $R$. The next two theorems contain our main results.

THEOREM 3.1. The following statements are equivalent for a space Z:

(a) $Z$ is pseudocompact.

(b) For all spaces $X, Y$ and all $g \in C(Z)$, and all $\phi: X \rightarrow Z, \alpha: Y \rightarrow Z$ having strongly-subclosed (mod $g$ ) graphs, $\mathcal{E}(\phi, \alpha, X \times Y, Z)$ is closed in $X \times Y$.

(c) Same as (b), except that $X=Y$.

(d) Same as (b), except that $X=Y$ and the conclusion is that $E(\phi, \alpha, X, Z)$ is closed in $X$. 
(e) Same as (b), except that $X=Y$ and the conclusion is that $E(\phi, \alpha, X, Z)$ $=X$ when $E(\phi, \alpha, X, Z)$ is dense in $X$.

Further, in (b), (c), (d) and (e), one can restrict $X$ to be $K$. In (b), (c), and (d), $\phi$ and $\alpha$ may both be restricted to be injective with $\phi(X)=\alpha(X)$. In (e), we may restrict either $\phi$ or $\alpha$ to be injective.

Proof that (a) Implies (b). Let $Z$ be pseudocompact, $g \in C(Z), \phi$ : $X \rightarrow Z$ and $\alpha: Y \rightarrow Z$ have strongly-subclosed (mod $g$ ) graphs, and let $(x, y)$ be a limit point of $\mathcal{E}(\phi, \alpha, X \times Y, Z)$. Let $\Omega$ be a filterbase on $\mathcal{E}(\phi, \alpha, X \times$ $Y, Z)-\{(x, y)\}$ with $\Omega \rightarrow(x, y)$. If $\pi_{X}$ and $\pi_{Y}$ represent the projections of $X \times Y$ onto $X$ and $Y$, respectively, it is easily seen that $\phi\left(\pi_{X}(F)\right)=\alpha\left(\pi_{Y}(F)\right)$ for each $F \in \Omega$. First suppose $\pi_{X}(F)=\{x\}$ for some $F \in \Omega$. Then we may assume without loss that $\pi_{Y}(\Omega)$ is a filterbase on $Y-\{y\}$. Since $\pi_{Y}(\Omega) \rightarrow y$ we have $\operatorname{ad}\left(\pi_{Y}(\Omega)\right)(\bmod g) \subset\{\alpha(y)\}$. We see readily that $\{\phi(x)\} \subset$ $\operatorname{ad} \phi\left(\pi_{X}(\Omega)\right)(\bmod g)=\operatorname{ad} \alpha\left(\pi_{Y}(\Omega)\right)(\bmod g)$. Hence $\phi(x)=\alpha(y)$ and $(x, y)$ $\in \mathcal{E}(\phi, \alpha, X \times Y, Z)$. A similar argument applies if $\pi_{Y}(F)=\{y\}$ for some $F \in \Omega$. In the remaining case, we may assume without loss that $\pi_{X}(\Omega)$ and $\pi_{Y}(\Omega)$ are filterbases on $X-\{x\}$ and $Y-\{y\}$, respectively. Since $\pi_{X}(\Omega) \rightarrow x$ and $\pi_{Y}(\Omega) \rightarrow y$ and $Z$ is pseudocompact we have $\varnothing \neq \operatorname{ad} \alpha\left(\pi_{Y}(\Omega)\right)(\bmod$ $g) \subset\{\alpha(y)\}$. Since ad $\alpha\left(\pi_{Y}(\Omega)\right)(\bmod g)=\operatorname{ad} \phi\left(\pi_{X}(\Omega)\right)(\bmod g) \subset\{\phi(x)\}$ we get $\phi(x)=\alpha(y)$ and $(x, y) \in \mathcal{E}(\phi, \alpha, X \times Y, Z)$.

Proof that (b) IMPLIES (c). Obvious.

Proof that (c) Implies (d). With $X=Y$ in (c) we see that $\mathcal{E}(\phi, \alpha, X \times$ $X, Z) \cap \Delta$ is closed in $\Delta$. Since the restriction of $\pi_{X}$ to $\Delta$ is a homeomorphism and $E(\phi, \alpha, X, Z)=\pi_{X}(\mathcal{E}(\phi, \alpha, X \times X, Z) \cap \Delta)$, (d) is verified.

Proof that (d) Imples (e). Since $E(\phi, \alpha, X, Z)$ is closed in $X$ from (d), and $E(\phi, \alpha, X, Z)$ is dense in $X$, we see that $E(\phi, \alpha, X, Z)=X$.

Proof that (e) implies (a). Let $g \in C(Z)$ and let $\Omega=\{F(1), F(2), \ldots\}$ be a strictly decreasing filterbase on $Z$ with ad $\Omega(\bmod g)=\varnothing$. Choose $x_{0}, y_{0} \in Z-F(1), x_{0} \neq y_{0}$, and for each $n=1,2, \ldots$, choose $x_{n} \in F(n)$ - $F(n+1)$. Let $X=\left\{y_{0}, x_{0}, x_{1}, \ldots\right\}$ topologized as follows: The open sets $A$ are those for which $y_{0} \notin A$ or $A \supset X \cap F(n)$ for some $n$. It is easy to see that $X$ is homeomorphic to $K$. Let $\phi: X \rightarrow Z$ be the identity function; and let $\alpha: X \rightarrow Z$ be defined by $\alpha(x)=x$ if $x \neq y_{0}$, and $\alpha\left(y_{0}\right)=x_{0}$. Then $E(\phi, \alpha, X, Z)=X-\left\{y_{0}\right\}$ which is dense in $X$. We will establish that $\phi$ and $\alpha$ have strongly-subclosed $(\bmod g)$ graphs to reach a contradiction. Let $y \in X$ and let $\Omega_{1}$ be a filterbase on $X-\{y\}$ with $\Omega_{1} \rightarrow y$. Then $y=y_{0}$ and $\Omega_{1}$ is finer than $\Omega$. So ad $\phi\left(\Omega_{1}\right)(\bmod g)=\operatorname{ad} \alpha\left(\Omega_{1}\right)(\bmod g) \subset \operatorname{ad} \Omega(\bmod g)=\varnothing$. So $\phi$ and $\alpha$ have strongly-subclosed $(\bmod g)$ graphs. The proof that (e) implies (a) is complete. The following construction may be used to show that $X$ may be restricted to be $K$, and that $\phi$ and $\alpha$ may be restricted to be injective with $\phi(X)=\alpha(X)$ in showing that (b) implies (a). $X$ is constructed as in the proof of (e) implies (a). We define $\alpha: X \rightarrow Z$ by $\alpha\left(x_{0}\right)=y_{0}, \alpha\left(y_{0}\right)=x_{0}$, and $\alpha(x)=x$ otherwise and let $\phi$ be the identity function. Then $\left(y_{0}, y_{0}\right) \in$ 
$\operatorname{cl}(\mathcal{E}(\phi, \alpha, X \times X, Z))-\mathcal{E}(\phi, \alpha, X \times X, Z)$. We establish that $\phi$ and $\alpha$ have strongly-subclosed (mod $g$ ) graphs as in the proof of (e) implies (a). Similar constructions will verify the remaining statements in the theorem.

The proof is complete.

The next and final theorem of this paper is established separately as it does not appear to fit easily into the scheme of proof used in Theorem 3.1.

3.2. Theorem. $A$ necessary and sufficient condition for a space $Z$ to be pseudocompact is that for all $g \in C(Z)$ and all spaces $X, \mathcal{E}(\phi, \phi, X \times X, Z)$ is a closed subset of $X \times X$ for all functions $\phi: X \rightarrow Z$ with strongly-subclosed (mod g) graphs.

Proof. Necessity. This follows directly from equivalence (c) of Theorem 3.1.

Sufficiency. Suppose $g \in C(Z)$ and suppose $\Omega=\{F(1), F(2), \ldots\}$ is a strictly decreasing filterbase on $Z$ with ad $\Omega(\bmod g)=\varnothing$. Let $X$ be the set constructed in the proof that (c) implies (a) of Theorem 3.1, topologized as follows: The open sets $A$ are those for which $A \cap\left\{x_{0}, y_{0}\right\}=\varnothing$ or $A \supset X \cap$ $F(n)$ for some $n$. Define $\phi: X \rightarrow Z$ by $\phi\left(x_{0}\right)=y_{0}, \phi\left(y_{0}\right)=x_{0}$ and $\phi(x)=x$ otherwise. Employing arguments similar to those above, we may establish that $\phi$ has a strongly-subclosed (mod $g)$ graph. If $W \in \Sigma\left(\left(x_{0}, y_{0}\right)\right)$ in $X \times X$, there is an $n$ such that $\left[\left(F(n) \cup\left\{x_{0}\right\}\right) \times\left(F(n) \cup\left\{y_{0}\right\}\right)\right] \cap(X \times X) \subset W$. Let $p \in F(n) \cap X$. Then $(p, p) \in W \cap \mathcal{E}(\phi, \phi, X \times X, Z)$. Hence $\left(x_{0}, y_{0}\right)$ $\in \operatorname{cl}(\mathcal{E}(\phi, \phi, X \times X, Z))-\mathcal{E}(\phi, \phi, X \times X, Z)$, a contradiction.

The proof is complete.

We close with the following remark.

3.3. REMARK. In each of our constructions in this section, we may add a discrete set of points onto the domains constructed and extend all functions, except for $\alpha$ in the proof that (c) implies (a) of Theorem 3.1, so that they are bijections. We simply define $\phi$ to map this added set in any one-to-one way onto $Z-\phi$ (domain of $\phi$ ) and let $\alpha(x)=\phi(x)$ for each $x$.

ACKNOWLEDGEMENT. I wish to thank the referee for a list of suggestions which was extensive and detailed; each suggestion resulted in an improvement in the quality of this paper.

\section{REFERENCES}

[BCM] R. W. Bagley, E. H. Connell and J. D. McKnight, Jr., On properties characterizing pseudocompact spaces, Proc. Amer. Math. Soc. 9 (1958), 500-506.

[C] W. W. Comfort, A nonpseudocompact product space whose finite subproducts are pseudocompact, Math. Ann. 170 (1967), 41-44. MR 35 \#965.

[F] Z. Frolik, The topological product of two pseudocompact spaces, Czechoslovak Math. J. 10 (1960), 339-349. MR 22 \# 7099.

[GJ] L. Gillman and M. Jerison, Rings of continuous functions, Van Nostrand, New York, 1960. MR 22 \#6994.

[G1] I. Glicksberg, Stone-C̈ech compactifications of products, Trans. Amer. Math. Soc. 90 (1959), 369-382. MR 21 \#4405. 
[G2] MR 14, 288.

[H] E. Hewitt, Rings of real-valued continuous functions. I, Trans. Amer. Math. Soc. 64 (1948), 45-99. MR 10, 126.

[J1] J. E. Joseph, Pseudocompactness via graphs and projections (submitted).

[J2] _ Multifunctions and graphs, Pacific J. Math. (to appear).

[SS] C. T. Scarborough and A. H. Stone, Products of nearly compact spaces, Trans. Amer. Math. Soc. 124 (1966), 131-147. MR 34 \# 3528.

[S] R. M. Stephenson, Jr., Pseudocompact spaces, Trans. Amer. Math. Soc. 134 (1968), 437-448. MR 38 \#674.

[T] R. Talamo, Pseudocompact spaces and functionally determined uniformities, Proc. Amer. Math. Soc. 56 (1976), 318-320.

Department of Mathematics, Howard University, Washington, D. C. 20059 